\title{
Internet of Things Based Monitoring and AI Based Testing Approach for Sensor Activity Testing of Embedded Systems
}

\author{
${ }^{* 1}$ Swapnili Karmore, ${ }^{2}$ Anjali Mahajan, ${ }^{3}$ Pravin Karmore \\ ${ }^{1}$ S.B.Jain Institute of Technology Management \& Research, \\ ${ }^{2}$ Government Polytechnic, \\ ${ }^{3}$ Shri Ramdeobaba College of Engineering and Management \\ Email: swapnilikarmore@sbjit.edu.in,armahajan@rediffmail.com,karmorepy@rknec.edu
}

\section{Received: 09 ${ }^{\text {th }}$ July 2018, Accepted: $14^{\text {th }}$ August 2018, Published: $3^{\text {st }}$ August 2018}

\begin{abstract}
The Internet of Things has been applied in many of the industries. Huge and large industries demand human less monitoring systems. Embedded system testing process proved as most critical and complex process in industries. In Embedded system testing, continues monitoring and testing of input, outputs and sensory activity is an essential requirement, each device is such typically equipped with a number of sensors, hence the multiple electronic control units are connected in order to provide control of the system. Normal Software testing is not suitable for embedded systems. Software as well as Hardware testing is required in case of embedded system, Testing of Embedded systems demonstrated through several simulation experiments. In this article, we propose small case studies for designing and development of testing framework via AI and IOT based approach, through extensive experiments testing of custom build an embedded system exhibits the variety of results proving its designing accuracy. The analysis is performed when data is generated synthetically as well as by actual developed embedded system. It also achieves increased efficiency, demonstrating the classification of valid and invalid data.
\end{abstract}

Keywords: Internet of Thing, Embedded Systems, Testing of Embedded System, and Artificial Intelligent based approach

\section{Introduction}

Now a day's Internet of thing is the most popular way of monitoring and managing the work associated in large industries. Embedded systems are known as the most demanding and a popular system, testing of embedded system's functionality is very complex and critical task. It includes real time environment monitoring in large buildings, medical equipment's functionality testing and accuracy in systems used in military and industry applications. Among several testing mechanism new approaches used for embedded system testing are Artificial Intelligence based. Intelligent Networks, that is responsible to monitor as well as to note the testing results of the systems.

An embedded system requires a number of sensors to indicate its different running states. Misbehavior of input mechanism can lead failure of output mechanisms. The sensors are responsible to show the behavior of the embedded system, whether the function which makes its safety critical are performing well or not. Artificial intelligence approach is used for testing of embedded system based on the classification problem used for nonlinear systems.

In this article, we propose Testing of embedded system via ANN. The main contributions based on this research work, can be summarized as Internet of Thing based approach for handling critical areas in industries. The Embedded system is designed in order to test its functionality. The ANN is used here for Testing of Embedded systems. Instead of testing software or hardware the function which makes its safety critical is tested here. The new approach is used for testing of embedded systems. The custom build embedded system is developed in order to perform testing on it. The embedded system is named as the oil level detection system. The function of this system is to measure the oil level inside large tanks, situated in the building.

The objective is to provide improved oil level detection processes, which will work only when a check of the system is desired. It is economical in cost to manufacture. It is simple and easy to use. Use of the Internet of things makes the system easy to operate and monitor from anywhere, in the absence of human observers.

\section{Materials and Methods:}

The new approach base on Artificial intelligence is used to solve the classification problem. Valid, invalid sets of inputs and outputs. To implement and support the concept, several research papers are studied. One of the researchers divides the methodology into the three important parts, first one is the covering interface second with system design and information processing and the second is optical fibre network framework and last is sensing modules having covered applications based on electronic sensors [1].

For oil level detection system, continuous monitoring is required. The liquid material filled with huge tank in large industries is difficult to measure. Embedded systems which involve in real time monitoring system is known as safety critical systems. Another researcher uses ultrasonic instrument for liquid level measurement of container which is sealed condition [2].

The researcher develops new method to measure and monitor the liquid level of huge tank. Monitoring 
and control were provided by RFID Tags. Energy efficient passive UHF RFID tags were used as level detector. The developed system consists of a storage tank, level sensors, passive tags and control circuits. The system is made-up of large pumps, huge storage tanks and passive tags for level sensors. Alarm circuit having based on the tag and indicator circuit was used for level detection [3].

In other literature optical technique is used for the continuous measurement of volume and liquid of transparent liquid in packed container, having cylindrical shape. This technique has several advantages over traditional measurement [4]. Another researcher introduces the novel structure for liquid level measurement. The working principle of this method is based on laser triangulation. Real time online measurement of the liquid surface can be performed via changing height of liquid surface in the tank [5].

Proposed work contains design and development of the custom build system.

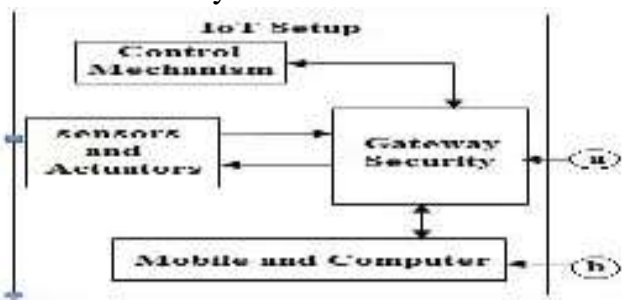

Fig 1: Block Architectural Diagram for IoT set up System Module-1

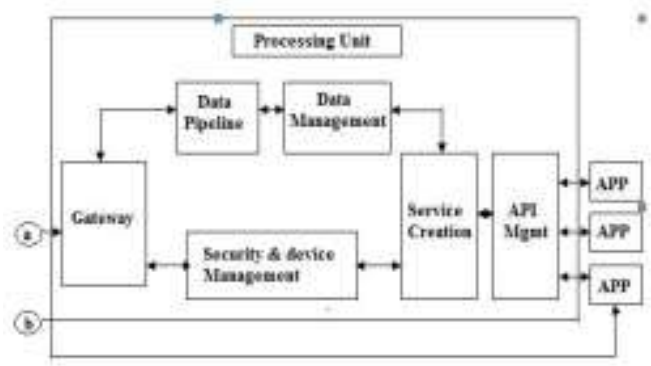

Fig 2: Block Architectural Diagram for Processing unit System Module-2

The system consists of IoT setup, which was responsible to monitor the several applications at a time. Processing unit contains data, security and API management. The IoT setup contains dashboard toward admin side. Admin can monitor status of all the applications in real time. Applications mentioned here were explained in detail named as oil level detection testing system. The system selected for testing is an oil level detection system in the industries, where the tank is situated on the top floor or where it can't be observed by a human being because of its larger size. If the level detection of tank fails the modules which will connect to perform important functions will also miss functions. Tanks having level detector sensors the sensors are connected to ADC; $\mathrm{ADC}$ is again connected to Microcontroller. Figure 1 and figure 2 show block architectural diagram of the overall system. Internet of Things based setup and processing unit are two important modules in the proposed system. Internet of thing based setup provides for oil level monitoring. The processing unit is responsible for data management.

Three Oil level detection system testing applications are connected to processing unit and Internet of Thing setup. Oil level detection testing system Internet of Things set up is responsible to monitor process and display data on the control panel and android based application.

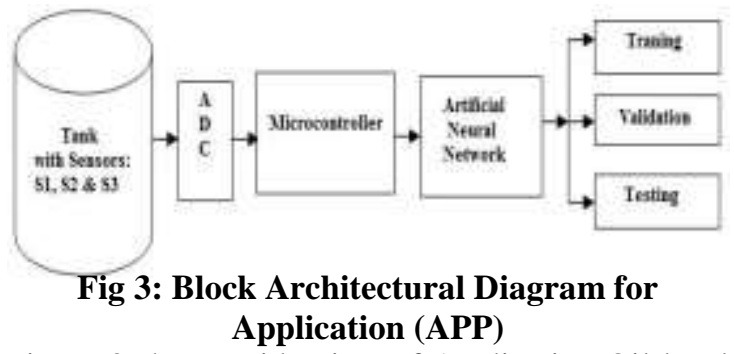

Figure 3 shows wide view of Application Oil level detection system. Such type of several applications was attached to Plant. This system includes level detector sensors which give the classified data generated by hardware. GUI (graphical user interface) is used for the Huge Tanks information and to check the results of Level sensors. The Huge Tank information on the Level is shown in the GUI as well as the data is created in a text file. This file is again classified for the next process which is fed through the artificial neural network.

The output is observed through MATLAB simulation misbehavior of input mechanism can lead failure of output mechanisms. The sensors are responsible to show the behavior of the embedded system, whether the function which makes its safety critical are performing well or not. Artificial intelligence approach is used for testing of embedded system based on the classification problem used for nonlinear systems.

Height of tank is fixed at 150 inches. Three sensors are attached to the wall of tank to detect the oil level depending on six types of conditions. The sensor is denoted as S1, S2 and S3, conditions are stated as follows:

If S1, S2, S3>127 Tank is full, labelled as Class 1 If S1, S2, S3<127 Tank is Empty, labelled as Class 2.If $\mathrm{S} 1, \mathrm{~S} 2>127 \& \mathrm{~S} 3<127$ Tank is partially empty, labelled as Class 3 or S2, S3>127 \& S1<127 Tank is partially empty or S1, S3, S7>127 S2<127 Tank is partially empty or $\mathrm{S} 1>127 \& \mathrm{~S} 2, \mathrm{~S} 3<127$ Tank is partially empty. Input data set will lies in the following distribution of classes.

\begin{tabular}{|l|l|l|l|}
\hline Class 1 & $111 \ldots 1$ & & \\
\hline Class 2 & & $111 \ldots 1$ & \\
\hline Class 3 & & & $111 \ldots 1$ \\
\hline
\end{tabular}

Table 1: Types of Classes 
Several experiments were conducted using artificial neural network and approaches for testing of embedded systems. In case of embedded system software embedded in hardware plays an important role in order to perform a particular function. While performing experiments we have considered test inputs and monitor the sensor activity of the system.

\begin{tabular}{|c|c|c|c|c|}
\hline S1 & S2 & S3 & Class & Pass/Fail \\
\hline 49.5304 & 2.14189 & 37.6558 & 2 & $\mathrm{P}$ \\
\hline 26.2389 & 101.346 & 45.4213 & 2 & $\mathrm{P}$ \\
\hline 68.8832 & 71.3478 & 125.411 & 3 & $\mathrm{~F}$ \\
\hline 109.57 & 64.412 & 104.672 & 3 & $\mathrm{~F}$ \\
\hline 122.148 & 32.0252 & 31.6488 & 2 & $\mathrm{P}$ \\
\hline 117.256 & 67.8075 & 82.5562 & 3 & $\mathrm{~F}$ \\
\hline 76.5374 & 76.7576 & 124.524 & 3 & $\mathrm{~F}$ \\
\hline 100.745 & 74.0538 & 94.1045 & 2 & $\mathrm{P}$ \\
\hline 120.215 & 82.8051 & 100.245 & 3 & $\mathrm{~F}$ \\
\hline 57.5672 & 104.047 & 35.7733 & 2 & $\mathrm{P}$ \\
\hline
\end{tabular}

Table 2: Types of Test Cases

The table shows the variety of test cases passed to the system. A valid and invalid set of data was passed to the Microcontroller via an interface for serial to parallel conversion. PIC Microcontroller was used to accept data from sensors attached to tank S1, S2 \& S3. AVR Microcontroller acts as an interface between level detection system and neural network. Back propagation and LM algorithm was used for ANN.

\begin{tabular}{|c|c|c|c|c|}
\hline Inputs & Class & Iteration & Test & Error Rate \\
\hline $\begin{array}{c}\text { S1- } 49.53 \\
\text { S2 -2.141 } \\
\text { S3-37.65 }\end{array}$ & 2 & 2 & Pass & Correct \\
\cline { 2 - 5 } & 4 & Fail & Not correct \\
\hline
\end{tabular}

Table 3: Observation

\section{Results and Discussion}

The main objective behind designing this section is a graphical calculation of results obtained in the overall research work. This section also mentions the performance indicators on the basis of research work carried out. The research work is developed based on multidisciplinary approach based on Internet of Things and Embedded System testing using Artificial Neural Network. Internet of things contains user side interface as an android application in mobile and dashboard on the admin side. Admin is responsible to monitor and provide control of data provided to Oil level detection system. Error obtained with the LM algorithm was less than backpropagation; there is no need of updating of weight, when we compared this error to the backpropagation algorithm, we got a less error in the first attempt. The initial computational parameter, i.e. $\delta$ is equal to the slope of the output.

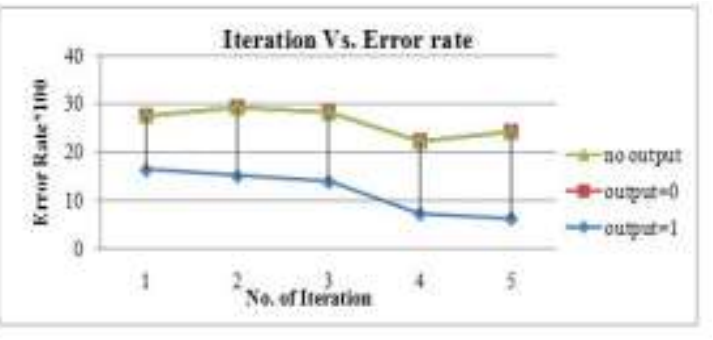

Fig 4: Iteration verses Error Rate in BP.

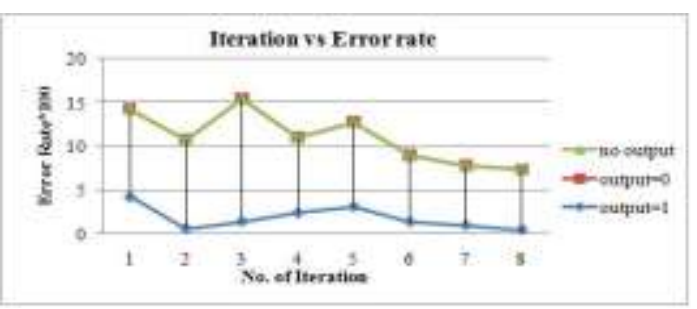

Fig 5: Iteration verses Error Rate in LM

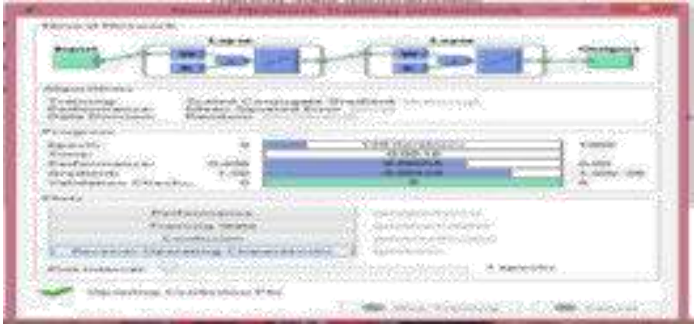

Fig 6: Neural Network for Training by NN tool

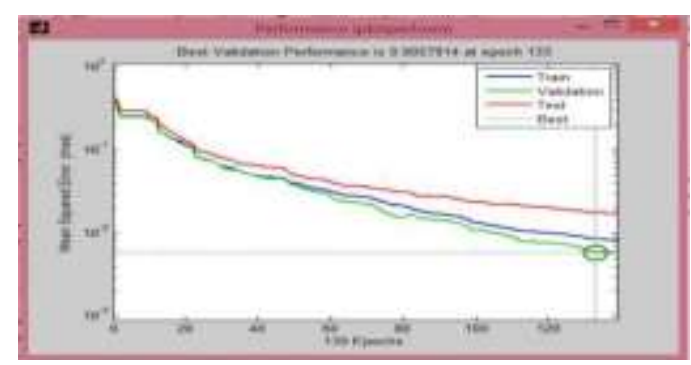

Fig 7: Performance Plot

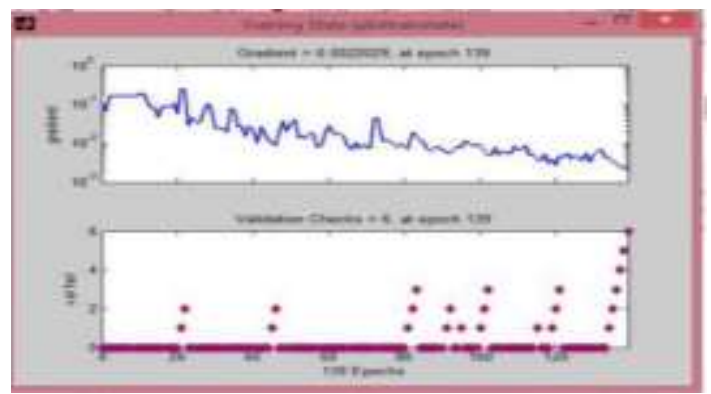

Fig 8: Training State

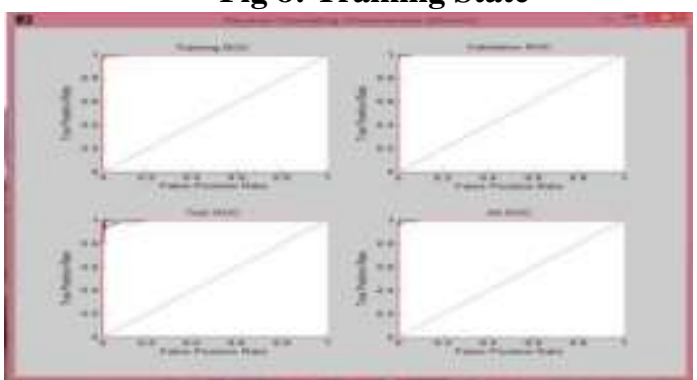

Fig 9: Receiver Operating State 


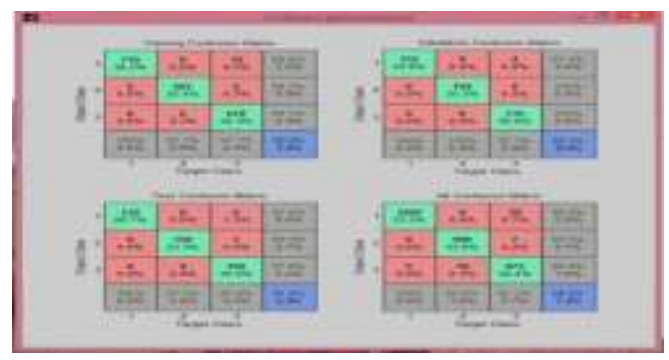

Fig 10: Confusion Plot

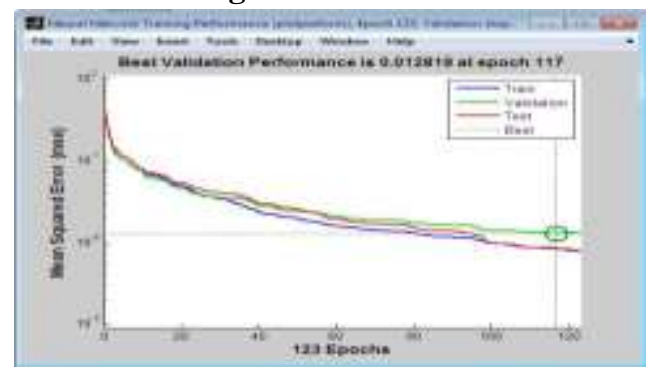

Fig 11: Output Version 2 Performance Plot

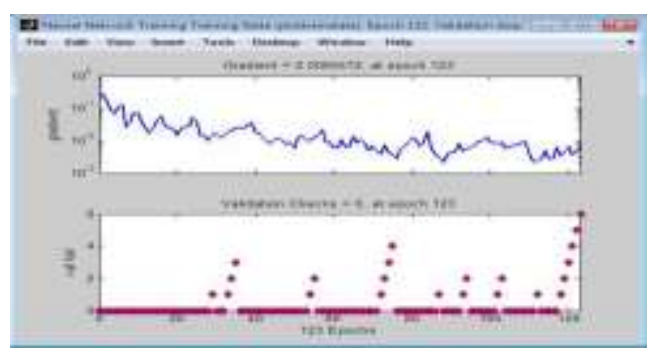

Fig 12: Training State Plot

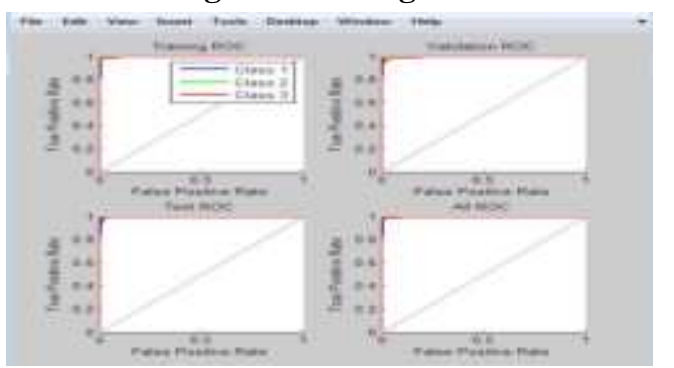

Fig 13: Receiver Operating Characteristic

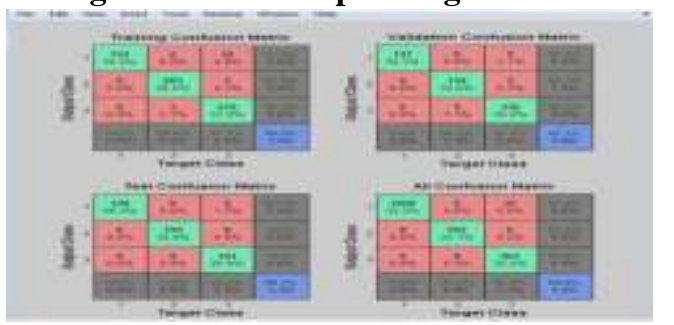

Fig 14: Confusion Plot

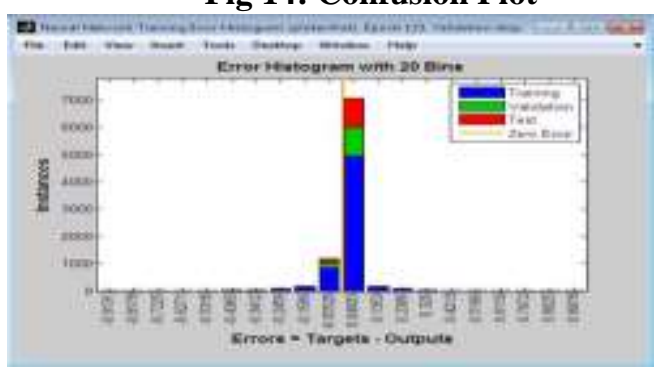

Fig 15: Error Histogram
Once the data is sorted as valid and invalid set of inputs, only valid inputs are passed to the system to avoid failure of functioning of the system. The above screenshot shows detailed result of research work obtained via ANN, where $70 \%$ of data is utilized for training $15 \%$ of data utilized for validation and 15 $\%$ of data utilized for testing.

\section{Conclusion}

The work described in this research is monitored via Internet of Things. The dashboard is developed for Admin side and android based application is developed toward User side. The Embedded system, Oil level detection system testing is performed via ANN. In the version one the overall efficiency obtained from training is $99 \%$, testing $98.1 \%$ and validation is $97.1 \%$ in the version two. From the above results the overall efficiency obtained from training is $99 \%$ Testing $99 \%$ and validation is $97.6 \%$. From the table and the dataset value it is observed. The safety critical function, accuracy its performance and error rate can be successfully calculated via LM algorithm. Classification of valid and invalid classes leads to avoid failure cases which improve efficiency of the overall system. Development of such environment avoids, use of real bulky, costly and complex embedded system for testing. Development of an artificial intelligence approach is competent enough to provide a common platform, where application specific and differential characteristics embedded systems can be tested successfully. Internet of things makes it human less platform independent system.

\section{References}

1. Muhammad Ammar Afif Ahmad, Izzat Syahmi Pauzi, "Smart Water Grade Continuous Monitoring Fiber Optic Sensing System”. 978-14799-8969-0/15/\$31.00 @2015 IEEE.

2. L. Chen, X. Dong, J. Han and P. Ye

"Development of a ultrasonic instrument for the sealed container's liquid level measurement". Proceedings of the Sixth World Congress on Intelligent Control and Automation (WCICA 2006), vol. 1, pp. 4972-4976, 2006.

3. C.-W. Lai, Y.-L. Lo, J.-P. Yur and C.-H. Chuang "Application of fiber brag grating level sensor and Fabry-Perot pressure sensor to simultaneous measurement of liquid level and specific gravity", vol. 12, no. 4, pp. 827-831, April, 2012.

4. H. Singh, S. Chakroborty, H. Talukdar, N. Singh and T. Bezboruah "A new non-intrusive optical technique to measure transparent liquid level and volume", vol. 11, no. 2, pp. 391-398, Feb., 2011.

5. H. Canbolat "A novel level measurement technique using three capacitive sensors for liquids", vol. 58, no. 10, pp. 3762-3768, Oct., 2009. 
6. W. Liu, G. Xie and L. Yang "Research on highprecision real-time online measurement of liquid level changes", Proceeding of the 2010 WASE International Conference on Information Engineering (ICIE), vol. 1, pp. 107-110.

7. C. P. Nemarich "Time domain refiectometry liquid levels sensors", vol. 4, no. 4, pp. 40-44, 2001.

8. M. Gerding, T. Musch and B. Schiek "A novel approach for a high-precision multi target-level measurement system based on time-domain reflectometry", vol. 54, no. 6, pp. 2768-2773, june, 2006.

9. E. Piuzzi, A. Cataldo and L. Catarinucci "Enhanced reflectometry measurements of permittivity and levels in layered petrochemical liquids using an 'in-situ' coaxial probe Measurement”, vol. 42, no. 5, pp. 685-696, 2009.

10. H. Zhuang, K.-S. Low and W.-Y. Yau, "A Pulsed Neural Network with On-Chip Learning and Its Practical Applications", IEEE Transactions on Industrial Electronics, vol. 54, pp. 34-42, 2007.

11. Justyna Zander-Nowickal, Zhen Ru Dail, "Model Driven Testing of Real-Time Embedded Systems- From Object Oriented towards Function Oriented Development”, IFIP 17th Intern. Conf. on Testing Communicating Systems - Test Com 2005, Montreal, Canada, ISBN: 3- 540-26054-4, Mai 2005.

12. C. Reichmann; P. Graf; KD. Mulier-Glaser: "GeneralStore - A CASETool Integration Platfonn Enabling Model Level Coupling of Heterogeneous Designs for Embedded Electronic Systems", 11th IEEE Conference on the Engineering of Computer Based Systems 2004, Brno, Czech Republic, May 2004.

13. Hua-ming Qian; Chun Zheng A, "Embedded Software Testing Process Model Computational Intelligence and Software Engineering", CISE International Conference on 2009.

14. Gang Liu, Qun Liu, Peng Xie, "Model-Based Testing and Validation on Knowledge-based Systems", IEEE International Conference Grey Systems and Intelligent Services, November 18-20, 2007, Nanjing, China.

15. Bart Broekman, Edwin Notenboo, "Testing Embedded Software", Text Book, Pearson publication, 2004.

16. Alper Sen, Etem Deniz, "Verification Test for Multicore Communication API. (MCAPI)", 2012. 12th International Workshop on Microprocessor Test and Verification, 2011. 